\title{
Numerical modelling of wind effects on breaking waves in the surf zone
}

\author{
Zhihua Xie ${ }^{1,2,3}$ (D)
}

Received: 2 February 2017 / Accepted: 5 July 2017 / Published online: 28 July 2017

(C) The Author(s) 2017. This article is an open access publication

\begin{abstract}
Wind effects on periodic breaking waves in the surf zone have been investigated in this study using a two-phase flow model. The model solves the Reynoldsaveraged Navier-Stokes equations with the $k-\epsilon$ turbulence model simultaneously for the flows both in the air and water. Both spilling and plunging breakers over a 1:35 sloping beach have been studied under the influence of wind, with a focus during wave breaking. Detailed information of the distribution of wave amplitudes and mean water level, wave-height-to-water-depth ratio, the water surface profiles, velocity, vorticity, and turbulence fields have been presented and discussed. The inclusion of wind alters the air flow structure above water waves, increases the generation of vorticity, and affects the wave shoaling, breaking, overturning, and splash-up processes. Wind increases the water particle velocities and causes water waves to break earlier and seaward, which agrees with the previous experiment.
\end{abstract}

Keywords Breaking waves - Wind-wave interaction - Surf zone $\cdot$ Two-phase flow model $\cdot$ Reynolds-averaged Navier-Stokes (RANS) equations · Volume of fluid method

Responsible Editor: Eric Deleersnijder

Zhihua Xie

zxie@cardiff.ac.uk

1 Hydro-environmental Research Centre, School of Engineering, Cardiff University, Cardiff, CF24 3AA, UK

2 Department of Chemical Engineering, Imperial College London, London, SW7 2AZ, UK

3 State Key Laboratory of Coastal and Offshore Engineering, Dalian University of Technology, Dalian 116024, China

\section{Introduction}

Wave breaking plays an important role in the air-sea interactions, surf zone dynamics, nearshore sediment transport, and wave-structure interactions. Over the last three decades, significant advances have been made in the theoretical, experimental and numerical studies of the characteristics of breaking waves (Peregrine 1983; Battjes 1988; Mei and Liu 1993; Banner and Peregrine 1993). However, little attention has been paid to investigate breaking waves under the influence of wind, either by experimental measurements or numerical simulations. When the wind is blowing over water waves, it can not only enhance the exchanges of heat, mass, and momentum on the air-water interface, but also affect the wave breaking process.

When the wind is absent, many research have been done in the past for better understanding of breaking waves. Much of our knowledge of breaking waves comes from laboratory measurements. Several systematic studies have been carried out for steady breaking waves (Duncan 1983), quasi-steady breaking waves (Lin and Rockwell 1995), unsteady deepwater breaking waves (Bonmarin 1989; Rapp and Melville 1990; Perlin et al. 1996; Tulin and Waseda 1999; Melville et al. 2002), and breaking waves in the laboratory surf zone (Nadaoka et al. 1989; Ting and Kirby 1994; Stansby and Feng 2005; Kimmoun and Branger 2007).

Some important effects of the wind on the breaking waves have been discussed in these works (Sobey 1986; Melville 1996; Jahne and Haussecker 1998; Dias and Kharif 1999). Banner and Phillips (1974) have considered the effect of a thin laminar wind drift layer in reducing the maximum wave height for deep-water breaking waves and found the "micro-breaking," which is important in the energy and momentum transfer from the wave to near surface turbulence and currents. Through the laboratory measurements 
for steady and unsteady breaking waves, Banner (1990) investigated the influence of wave breaking on the surface pressure distribution in wind-wave interaction and found that the form drag and wind stress increase during wave breaking. Ward et al. (1998) conducted a series of physical model tests to study the effects of onshore wind on run-up elevations. It was suggested that low wind speeds have little effect on run-up, but higher wind speeds significantly increase run-up elevations. Feddersen and Veron (2005) carried out a laboratory study of wind effects on shoaling wave shape over a steep beach (slope 1:8) in a laboratory wind wave tank and found that wind increases the shoaling wave energy and has a significant effect on the wave shape (e.g. changes wave skewness and asymmetry). Douglass (1990) has experimentally investigated the influence of local wind on nearshore breaking waves in a laboratory wind-wave flume and found that wind has significant effects on the breaker location, geometry, and type. Onshore winds cause waves to break earlier and in deeper water further from shore; offshore winds cause waves to break later and in shallower water closer to shore. He found that wind effect on breaker depth is significant while on breaker height is slight. Douglass (1990) indicated that primary mechanism for wind affecting breaking waves appears to be shear, not normal stress and concluded that "Surf zone dynamics models that ignore wind or include wind only as a surface shear may be missing a very important effect of the wind-its effect on the initiation and mechanics of wave breaking."

Many numerical models have been developed to simulate periodic breaking waves in the surf zone. One of these is the Boussinesq-type model (Madsen et al. 1997; Veeramony and Svendsen 2000; Lynett 2006), which is widely used in the nearshore wave modelling. With developments of CFD (computational fluid dynamics) and increases in computer power, recent models for studying free surface flows, including breaking waves, solve the Navier-Stokes equations coupled with a free surface calculation (see Lin 2008 for comprehensive modelling applications and methodologies for water waves). There are several numerical studies on breaking waves in the surf zone based on one-phase flow model (Lin and Liu 1998a; 1998b; Bradford 2000; Mayer and Madsen 2000; Zhao et al. 2004; Shao 2006; Shao and Ji 2006; Bakhtyar et al. 2009; Christensen and Deigaard 2001; Watanabe et al. 2005), in which only the flow in the water is considered in the computation, the pressure in the air is taken as a constant, and the boundary conditions are specified at the free surface. When the wind is present, the pressure in the air is no longer a constant and the previously used boundary conditions are not valid at the interface. In order to take the air into account for wave breaking, recently, several two-phase flow model (Hieu et al. 2004; Lubin et al. 2006; Wang et al. 2009; Lakehal and Liovic 2011; Xie 2012; 2013), in which both flows in the air and water are solved, have been developed to study the details of breaking waves in the surf zone and the air entrainment during wave breaking. The two-phase flows model is able to represent the wind field in a more accurate manner and provide velocity field and turbulence during wave breaking in much more detail. However, the effect of wind on breaking waves has not been explored in their studies.

Most flows in breaking waves are turbulent and therefore need different treatment for the turbulence. In most engineering applications, the Reynolds-averaged Navier-Stokes (RANS) approach is often employed as it requires less computational effort when compared to direct numerical simulation and large-eddy simulation. In RANS modelling, the $k-\epsilon$ model is one of the most widely used two-equation turbulence models. It has been tested over a large variety of flow situations and therefore its limitations, as well as its successes, have become well understood (Versteeg and Malalasekera 2007). The $k-\epsilon$ turbulence model has been successfully applied for a wide range of hydraulic problems (Rodi 1993) and surf zone dynamics (Lin and Liu 1998a; Bradford 2000).

In order to investigate the influence of wind on water waves, very few numerical studies have been carried out. Chen et al. (2004) implemented the parameterized wind stress into Boussinesq wave models to investigate the nearshore wave propagation and horizontal circulation and Liu et al. (2016) further developed this model by introducing a wind-source term based on a wave-induced pressure perturbation to study wind effects on triad nonlinear interaction. Kharif et al. (2008) applied an empirical wind pressure distribution on the free surface using Jeffreys sheltering theory (Jeffreys 1925) in their potential flow model, to calculate the influence of wind on extreme wave events. Yan and Ma (2010) used a potential flow model combined with a commercial software to investigate the interaction between wind and 2D freak waves. Xie (2014) studied wind effects on breaking solitary waves over a sloping beach. However, none of them study periodic breaking waves in the surf zone.

Thus, the objective of the present study is to investigate wind effects on periodic breaking waves in the surf zone and provide detailed information during wave breaking. A recently developed two-phase flow model (Xie 2012; 2015), which solves the flow in the air and water simultaneously, is employed here to study spilling and plunging breakers in the experiment of Ting and Kirby (1994) under the influence of wind. The description of the mathematical model for the two-phase flow is described in next section. The numerical method is presented after that. Both spilling and plunging breakers in the surf zone are simulated in the results and discussion section. Detailed computational results of the water surface profiles, velocity, vorticity, and turbulence fields are shown and discussed. Finally, conclusions are drawn at the end. 


\section{Mathematical model}

\subsection{Geometry}

Wind effects on two-dimensional periodic breaking waves along a uniform sloping beach of angle $\beta$ are considered in this study. The schematic of this problem is shown in Fig. 1, where the origin of the coordinate system is on the still water surface where the local still water depth $d(x)=d_{0}, x$ and $z$ are the horizontal and vertical coordinates, respectively, $H$ is the wave height, $d_{0}$ is the water depth in the horizontal region, $\zeta(x, t)$ is the water surface elevation, $t$ is the time, $\bar{\zeta}(x, t)$ is the mean water surface elevation, $d(x)$ is the local still water depth, and $h(x)=d(x)+\bar{\zeta}(x, t)$ is the local mean water depth.

\subsection{Governing equations}

The governing equations for incompressible Newtonian fluid flow are the Reynolds-averaged Navier-Stokes equations. Mass conservation is described by the continuity equation

$\frac{\partial \rho}{\partial t}+\nabla \cdot(\rho \boldsymbol{u})=0$

where $\rho$ is the density and $\boldsymbol{u}=(u, w)$ is the velocity vector.

If we assume that the fluid is incompressible $(\mathrm{d} \rho / \mathrm{d} t=$ 0 ), then the continuity equation can be simplified to

$\nabla \cdot u=0$.

The momentum conservation is expressed as

$\frac{\partial(\rho \boldsymbol{u})}{\partial t}+\nabla \cdot(\rho \boldsymbol{u} \otimes \boldsymbol{u})=-\nabla p+\nabla \cdot\left[\left(\mu+\mu_{t}\right)\left(\nabla \boldsymbol{u}+\nabla^{\mathrm{T}} \boldsymbol{u}\right)\right]+\rho \boldsymbol{g}$,

where $p$ represents pressure, $\boldsymbol{g}$ the gravitational acceleration vector, $\mu$ the dynamic viscosity of the fluid, and

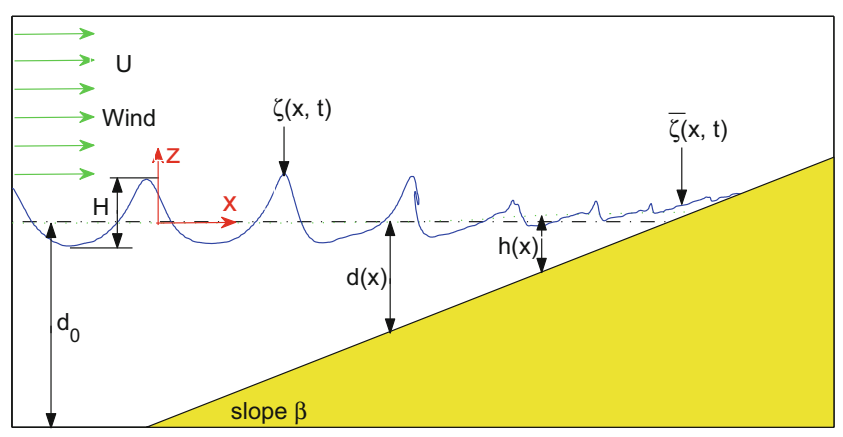

Fig. 1 The sketch of wind effects on periodic breaking waves in the surf zone (not scaled). $x$ and $z$ are the horizontal and vertical coordinates, respectively, $H$ is the wave height, $d_{0}$ is the water depth in the horizontal region, $\zeta(x, t)$ is the water surface elevation, $t$ is the time, $\bar{\zeta}(x, t)$ is the mean water surface elevation, $d(x)$ is the local still water depth, and $h(x)=d(x)+\bar{\zeta}(x, t)$ is the local mean water depth $\mu_{t}=\rho C_{\mu} k^{2} / \epsilon$ is the turbulent eddy viscosity. $k$ is turbulent kinetic energy and $\epsilon$ is turbulent eddy dissipation which are governed by the $k-\epsilon$ turbulence model (Launder and Spalding 1974)

$$
\frac{\partial(\rho k)}{\partial t}+\nabla \cdot(\rho \boldsymbol{u} k)=\nabla \cdot\left[\left(\mu+\frac{\mu_{t}}{\sigma_{k}}\right) \nabla k\right]+P_{k}-\rho \epsilon,
$$

$$
\frac{\partial(\rho \epsilon)}{\partial t}+\nabla \cdot(\rho \boldsymbol{u} \epsilon)=\nabla \cdot\left[\left(\mu+\frac{\mu_{t}}{\sigma_{\epsilon}}\right) \nabla \epsilon\right]+C_{1 \epsilon} \frac{\epsilon}{k} P_{k}-C_{2 \epsilon} \rho \frac{\epsilon^{2}}{k},
$$

where $P_{k}=\mu_{t}\left(\partial u_{i} / \partial x_{j}+\partial u_{j} / \partial x_{i}\right)^{2} / 2$ is the turbulent production term, and the empirical coefficients $C_{\mu}, \sigma_{k}, \sigma_{\epsilon}$, $C_{1 \epsilon}$, and $C_{2 \epsilon}$ are given in Table 1 .

The momentum equation is closed with the constitutive relations for the density and dynamic viscosity of the fluid

$\rho=F \rho^{\mathrm{w}}+(1-F) \rho^{\mathrm{a}}$,

$\mu=F \mu^{\mathrm{w}}+(1-F) \mu^{\mathrm{a}}$,

where the superscripts $\mathrm{w}$ and a denote fluid water and air, respectively. $F$ is the volume fraction defined as

$F=\left\{\begin{array}{l}1, \text { if only water is present; } \\ 0, \text { if only air is present. }\end{array}\right.$

The air-water interface is then within the cells where $0<$ $F<1$. A particle on surface stays on surface and the volume fraction $F$ has a zero material derivative

$\frac{\mathrm{d} F}{\mathrm{~d} t}=\frac{\partial F}{\partial t}+\boldsymbol{u} \cdot \nabla F=0$.

These equations complete the mathematical description of the two-phase flow model.

\subsection{Initial and boundary conditions}

At the inlet, the cnoidal wave is generated by specifying the water surface elevation and water particle velocities based on the third-order cnoidal wave theory (Horikawa 1988) and more details can be found in Xie (2013). The effect of wind is obtained by specifying uniform wind speed above the maximum water surface elevation. Only the onshore wind $U$ is considered in this study although the offshore wind effect can be obtained in a similar way. We assume development time for the boundary layer is negligible and for the sake of simplicity, only uniform wind profiles are considered here although other more physically realistic wind profiles can also be studied. It is worth mentioning that a ramping

Table 1 Empirical coefficients in the $k-\epsilon$ turbulence model

\begin{tabular}{ccccc}
\hline$C_{\mu}$ & $\sigma_{k}$ & $\sigma_{\epsilon}$ & $C_{1 \epsilon}$ & $C_{2 \epsilon}$ \\
\hline 0.09 & 1.0 & 1.3 & 1.44 & 1.92 \\
\hline
\end{tabular}


function is applied to the water surface elevation and velocities during the first wave period, so that the flow will change slowly when the computation starts. For the turbulence field, the method of Lin and Liu (1998a) is adopted here. The turbulent kinetic energy is obtained as $k=(I \times C)^{2} / 2$, where $C$ is the wave phase speed and $I$ is the turbulent intensity. The turbulent eddy dissipation $\epsilon=\rho C_{\mu} k^{2} /\left(I_{\epsilon} \times \mu\right)$ is adjusted so the turbulent eddy viscosity is $I_{\epsilon}$ times the dynamic viscosity of each fluid at the inlet. The same values in Lin and Liu (1998a) are used in this study: $I=0.0025$ and $I_{\epsilon}=0.1$.

At the outlet, the zero gradient boundary conditions are applied for the mean flow and turbulence fields.

The bottom and top of the domain and the sloping beach are considered as solid boundaries and the wall function method is used to model the near-wall region.

At $t=0$, the simulations are started from a quiescent state to let the flow develop in time. The water surface is given as the initial still water depth $d(x)$, from which the volume fraction in each cell is calculated. The velocity fields in both air and water are initialized as zero. The hydrostatic pressure distribution is applied in the whole domain depending on the local density and height. The turbulence field ( $k$ and $\epsilon$ ) is initialized to the same value as the boundary conditions at the inlet mentioned above.

\section{Numerical method}

For the sake of brevity, only a brief description of the numerical method is presented here, and more details of the numerical method for the two-phase flow model can be found in previous works (Xie 2010; 2012; 2015). In this study, the governing equations are discretised using the finite volume method on a staggered Cartesian grid. The advection terms are discretised by a high-resolution scheme (Hirsch 2007), which combines the high order accuracy with monotonicity, whereas the gradients in pressure and diffusion terms are obtained by central difference schemes. In order to deal with complex geometries in Cartesian grids, the partial cell treatment (Torrey et al. 1985) is utilised in the finite volume discretisation, in which the advective and diffusive fluxes at cell faces as well as cell volume are modified in cut cells. The PISO algorithm (Issa 1986) is employed in the present study for the pressure-velocity coupling and a backward finite difference discretisation is used for the time derivative, which leads to an implicit scheme for the governing equations. In this study, the air-water interface is captured by a high-resolution VOF scheme CICSAM (Compressive Interface Capturing Scheme for Arbitrary Meshes) (Ubbink 1997), which does not need to reconstruct the interface after each time step. The present model has been validated against available experimental data for overturning waves over a sloping beach and a reef (Xie 2012), periodic breaking waves in the surf zone (Xie 2013), breaking solitory waves over a slope (Xie 2014) and breaking waves over complex topography (Xie 2015), in which the overturning jet, air entrainment and splash-up have been captured during wave breaking .

\section{Results and discussion}

In this section, we present the numerical results of wind effects on spilling and plunging breakers in the surf zone. The experiment of Ting and Kirby (1994) is considered here, which is widely used by several numerical studies for breaking waves in the absence of wind. Water surface profiles, mean velocity, vorticity, and turbulent kinetic energy fields during wave breaking under the influence of wind are shown and discussed.

\subsection{Computational setup}

In the simulation, the computational model is set up to replicate the laboratory model studies undertaken and reported by Ting and Kirby (1994) as shown in Fig. 1, where the origin of the coordinates is located at the still water level at which the local still water depth is $0.38 \mathrm{~m}$, the beach slope is $1 / 35$ and $d_{0}=0.4 \mathrm{~m}$ is the water depth in the horizontal region. Cnoidal waves are generated at the inlet on the left of the domain, the sloping beach, bottom and top of the domain are considered as solid boundaries while the right of the domain is the outlet. A key parameter for this problem is the ratio of wind speed $U$ to wave phase speed $C$. Only the onshore wind $U / C$ in the range of 0 to 2 is considered in this study although the offshore wind effect can be obtained in a similar way. The computational domain (started from $x=-4.3 \mathrm{~m}$ in the $x$ direction and $y=-0.4$ $\mathrm{m}$ in the $y$ direction) is $22 \mathrm{~m}$ long and $0.8 \mathrm{~m}$ high, and it is discretised by a uniform grid with $\Delta x=0.02 \mathrm{~m}$ and $\Delta z=0.008 \mathrm{~m}$, which is similar to the previous numerical studies (Bradford 2000; Zhao et al. 2004) in the absence of wind. In order to get the detail during wave breaking, the time step is automatically adjusted with a maximum value $0.2 \Delta t^{\mathrm{CFL}}$ during the simulation, where $\Delta t^{\mathrm{CFL}}$ is the time step to satisfy the Courant-Friedrichs-Lewy (CFL) stability criterion. The computations are run up to $50 \mathrm{~s}$ for spilling breakers and $60 \mathrm{~s}$ for plunging breakers, and the period of the last five waves are used to obtain the mean value for the analysis. Table 2 shows the wave conditions for the spilling and plunging breakers in the experiment in the absence of wind, where $T$ is the wave period, $H_{0}$ and $L_{0}$ are the wave height and wave length in deep water, and $x_{b}$ and $d_{b}$ are the location and water depth of wave breaking. 
Table 2 Wave conditions in the experiment of Ting and Kirby (1994)

\begin{tabular}{lllllll}
\hline $\begin{array}{l}\text { Breaker } \\
\text { type }\end{array}$ & $\begin{array}{l}H_{0} \\
(\mathrm{~m})\end{array}$ & $\begin{array}{l}H \\
(\mathrm{~m})\end{array}$ & $\begin{array}{l}T \\
(\mathrm{~s})\end{array}$ & $H_{0} / L_{0}$ & $\begin{array}{l}x_{b} \\
(\mathrm{~m})\end{array}$ & $\begin{array}{l}d_{b} \\
(\mathrm{~m})\end{array}$ \\
\hline Spilling & 0.127 & 0.125 & 2.0 & 0.02 & 6.400 & 0.196 \\
Plunging & 0.089 & 0.128 & 5.0 & 0.0023 & 7.795 & 0.156 \\
\hline
\end{tabular}

The subscripts 0 and $\mathrm{b}$ denote deep water and breaking point

\subsection{Spilling breakers}

In this section, we first investigate the wind effects on spilling breakers. Results for three cases when $U / C=0$, $U / C=1$, and $U / C=2$ are shown and discussed.

Figure 2 shows the wind effects on the distribution of wave amplitudes and mean water level (top) and the effect of wind on the wave-height-to-water-depth ratio across the surf zone (middle). It can be seen that the numerical results for $U / C=0$ agree well with the experimental measurements in the absence of wind. In the presence of wind, during wave shoaling, the maximum wave elevation is very similar for all case $(0.1215,0.1225$, and 0.1204 for $U / C=0,1,2)$, but occurs at different locations. During wave breaking, it
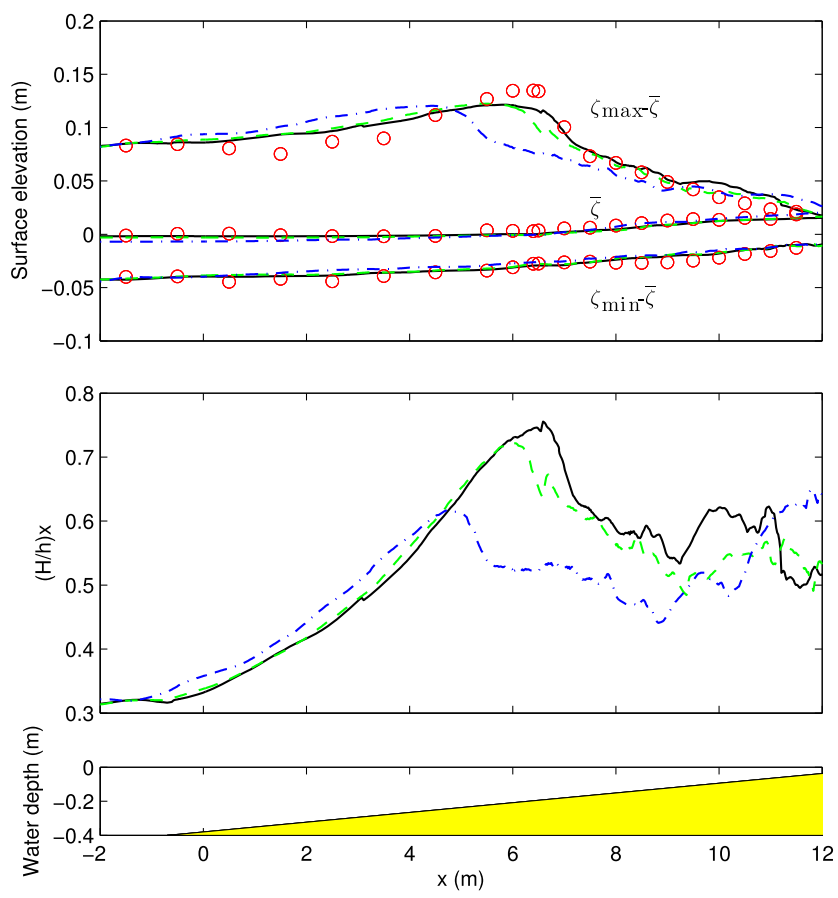

Fig. 2 Wind effects on the distribution of maximum and minimum wave elevation and mean water level (top) and the wave-height-towater-depth ratio (middle) for the spilling breaker case on the sloping beach for the experiment of (Ting and Kirby 1994) (bottom). Red circles are experimental data (Ting and Kirby 1994) in the absence of wind; black solid lines: $U / C=0$; green dashed lines: $U / C=1$; blue dash-dotted lines: $U / C=2$ can be seen from Fig. 2 that the wave breaks earlier and further from shore when the wind speed $U / C$ increases, which is consistent with the previous laboratory studies by Douglass 1990 and King and Baker (1996). This is attributed to the direct push of the wind and the increased kinetic energy obtained from the wind in water waves. The water particle velocity is increased by the wind and thus the wave breaks earlier. This phenomenon has also been observed in the study of wind effects on breaking solitary waves in Xie (2014). After wave breaking in the bore region, the wind has little effect on the crest and trough level of the wave. It is shown from Fig. 2 that the mean water level has been changed by the wind. In the surf zone, the radiation stress (Longuet-Higgins and Stewart 1962) increases before the breaking point due to the decreasing water depth and decreases after the breaking point due to the energy dissipation. Therefore, through the horizontal momentum flux balance, the slope of the mean water level, namely, the wave set-up and set-down, are related to the change of radiation stress across the surf zone (Sorensen 2006). In the presence of wind, the slope of the mean water level increases when the wind speed $U / C$ increases from 0 to 2 , which is attributed to wind forcing. It can also be seen from Fig. 2 that during wave breaking, the wind effect on the wave height is slight but the wind thus affect what water depth breaking occurs, thus the effect of wind on the breaker height-to-depth ratio is significant, which is consistent with the previous laboratory studies by Douglass (1990). The ratio during wave breaking decrease with the increase of wind speed, which has important implications in some surf zone dynamics models.

In order to investigate the wind effects on the velocity field during wave breaking, Fig. 3 shows the velocity fields and water surface profile for the spilling breaker in one wave period at $t / T=0.0,0.1,0.2,0.4$ with $(U / C=2)$ and without $(U / C=0)$ wind, where $t / T=0.0$ corresponds to when the wave is close to the breaking point. At $t / T=0.0$, the front of the wave becomes nearly vertical and the velocity in the water is slightly smaller than the wave phase speed. It can be see that the air flow has been changed significantly due to wind forcing between two cases. A recirculation of air flow can be easily seen on the top of the wave as the air is driven by the wave motion when $U / C=0$, while the recirculation of air flow does not exist with all air moving onshore instead. It is worth remarking that the wave is driven by the wind when $U / C=2$ while the air is driven by the wave when $U / C=0$. It can also be seen from Fig. 3 that the breaking point is well predicted in the model when compared with the experiment in the absence of wind. During wave breaking at $t / T=0.1$, an overturning jet is formed at the front face of the wave for both cases. Compared to the case $U / C=0$, the forming jet when $U / C=2$ is slightly smaller due to the wind shear. For both cases, the velocity 
Fig. 3 Velocity fields during wave breaking for the spilling breaker case at

$t / T=0.0,0.1,0.2,0.4$ when $\mathbf{a}$

$U / C=0$ (from Xie 2013) and

b $U / C=2$. Velocities are

normalized by the wave phase speed $C$. For clarity, only velocity vectors in every five points are shown here
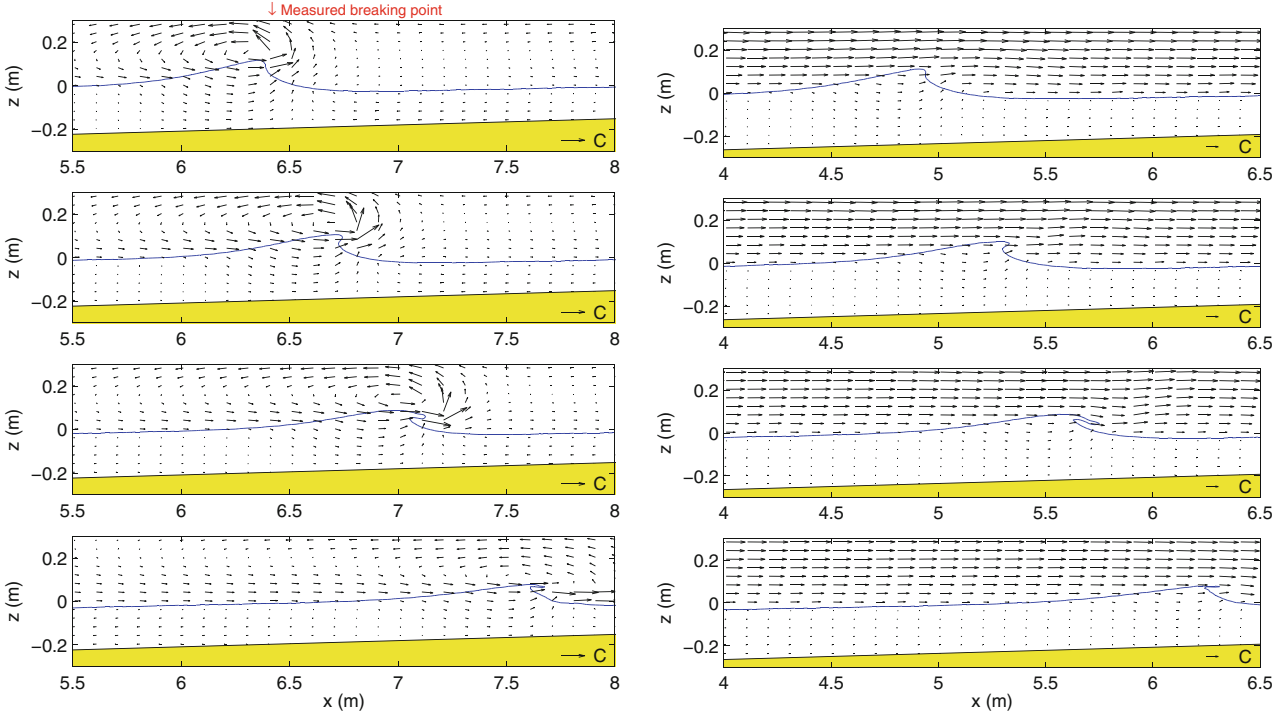

(a) $U / C=0$

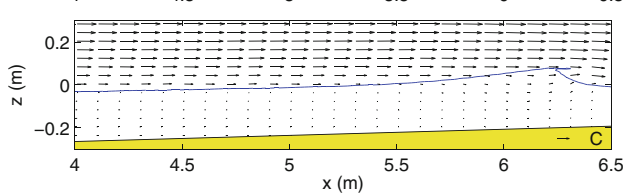

(b) $U / C=2$ in the water increases from the bottom to the water surface with the maximum velocity located on the tip of the overturning jet. During wave curling down at $t / T=0.2$, the developed jet becomes thinner under the influence of wind. It can be seen that in front of the overturning jet, the air is pushed by the wave when $U / C=0$ while the air follows the wind when $U / C=2$. At $t / T=0.4$, the overturning jet spills down the front face of the wave and similar profiles can be seen for both cases. It is worth noting that although similar wave breaking process is observed in the absence or in the presence of wind, the location of the breaking point has been changed significantly. The breaking point is about $x_{b}=6.4 \mathrm{~m}$ for $U / C=0$ but $x_{b}=4.95 \mathrm{~m}$ for $U / C=2$. It is shown that under the influence of wind, the wave breaks in deeper water and further from shore which is consistent with the previous laboratory studies by Douglass (1990) and King and Baker (1996).

Figure 4 shows the mean vorticity fields for the spilling breaker when $U / C=2$ in one wave period at $t / T=$ $0.0,0.1,0.2,0.4$. The vorticity in the water has a smaller value in comparison with the vorticity in the air. In the air, the vorticity field is totally different from that for $U / C=0$ with large positive vorticity above the wave crest due to the recirculation of air flow, which is shown in Xie (2013). However for $U / C=2$, there is no positive vorticity above the wave crest as the wind is moving onshore. Large negative vorticity is generated in the vicinity of the water surface due to the wind-induced shear. A small region of positive vorticity appears in front of the wave during wave breaking as the air tries to escape from the cavity. In the water, the vorticity generation during wave breaking is similar to that for $U / C=0$, except that the wave breaks in a deeper region.
In order to investigate the wind effects on the turbulence field during wave breaking, Fig. 5 shows the turbulent kinetic energy fields for the spilling breaker in one wave period at $t / T=0.0,0.1,0.2,0.4$ with $(U / C=2)$ and without $(U / C=0)$ wind. The turbulence is weak in the outer surf zone as the turbulence is primarily originated from the breaker. Once the wave breaks, the turbulent kinetic energy is high behind the breaking wave front, decreasing towards the back face of the wave and varying slowly with the water depth, which is consistent with experimental observation (Ting and Kirby 1994). In the presence of wind, it can be seen that the turbulence is suppressed as the wave breaks in a deeper region.

\subsection{Plunging breakers}

After studying spilling breakers, in this section, we investigate wind effects on plunging breakers. Results are shown for three cases when $U / C=0, U / C=1$ and $U / C$ $=2$.

Figure 6 shows the wind effects on the distribution of wave amplitudes and mean water level (top) and the effect of wind on the wave-height-to-water-depth ratio across the surf zone (middle). It can be seen that the numerical results for $U / C=0$ agree well with the experimental measurements in the absence of wind. In the presence of wind, there is nearly no difference for the evolution of minimum wave elevation in the whole region, while the evolution of maximum wave elevation has been changed significantly, which is similar to the case for spilling breakers. It is shown from Fig. 6 that the mean water level has been changed slightly by the wind. The mean water level decreases in the shoaling region and increases in the bore region with the increase of wind 
Fig. 4 Mean vorticity fields during wave breaking for the spilling breaker case when $U / C=2$ at

$t / T=0.0,0.1,0.2,0.4$. The vorticity has been normalized by $\sqrt{g /\left(d_{0}+a\right)}$, where

$a=\max [\zeta(x=-4.3, t)]-d_{0}$ is the incident wave amplitude at the inlet and the contours are shown for

$\pm[1,2.5,5,10,15,20,25]$

Fig. 5 Turbulent kinetic energy fields during wave breaking for the spilling breaker case at $t / T=0.0,0.1,0.2,0.4$ when a $U / C=0$ and $\mathbf{b} U / C=2$.

Turbulent kinetic energy are normalized by $C^{2}$
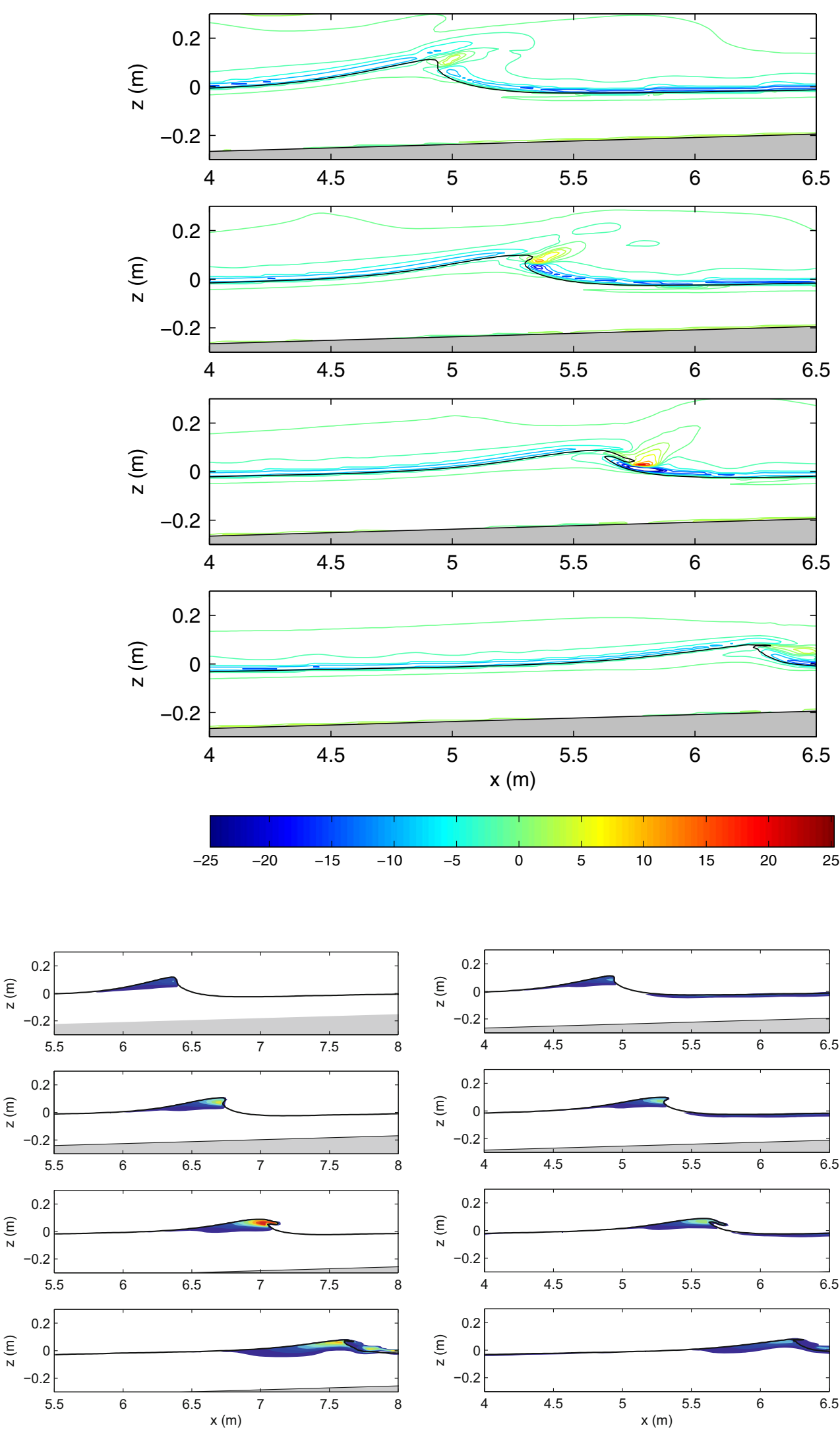

(a) $U / C=0$

(b) $U / C=2$ 

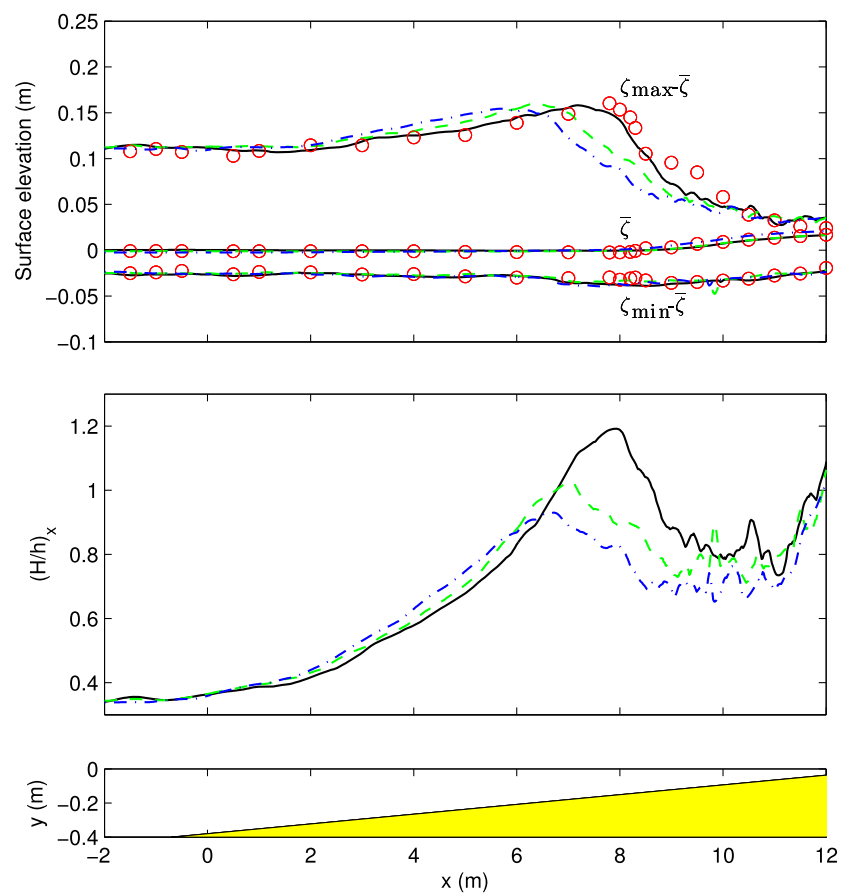

Fig. 6 Wind effects on the distribution of maximum and minimum wave elevation and mean water level (top) and the wave-height-towater-depth ratio (middle) for the plunging breaker case on the sloping beach for the experiment of Ting and Kirby (1994) (bottom). Red circles are experimental data (Ting and Kirby 1994) in the absence of wind; black solid lines: $U / C=0$; green dashed lines: $U / C=1$; blue dash-dotted lines: $U / C=2$

speed $U / C$. The driving mechanism for the change of the slope of the mean water level is similar to that discussed in the spilling breaker case (see Subsection 4.2). It can also be seen from Fig. 6 that breaker height-to-depth ratio decreases with the increase of wind speed as the wave breaks further offshore. Compared to spilling breakers, larger height-todepth ratio is observed in plunging breakers.

Figure 7 shows the velocity fields and water surface profile for the plunging breaker in one wave period at $t / T=0.0,0.05,0.1,0.2$ with $(U / C=2)$ and without $(U / C=0)$ wind, where $t / T=0.0$ corresponds to when the wave is close to the breaking point. Compared to the spilling breaker, stronger wave breaking (in terms of overturning water column) is observed in the plunging breaker before wave breaking $(t / T=0.0)$, during wave overturning $(t / T=0.05)$, during the splash-up $(t / T=0.1)$ and the jet-splash cycle $(t / T=0.2)$ processes. It is worth noting that the overturning jet curls down and impinges on the water ahead to generate the splash-up in plunging breakers, whereas the overturning jet only spills down the front face of the wave during wave breaking in spilling breakers. The jet-splash cycles only occur in the plunging breaker case. The wind effects on plunging breakers are similar to the spilling breaker case, where the air flow has been changed significantly due to wind forcing and the breaking point has been moved seaward. The breaking point is about $x_{b}=7.7 \mathrm{~m}$ for $U / C=0$ while $x_{b}=6.25 \mathrm{~m}$ for $U / C=2$. It is noted that the breaking point has been shifted about $1.5 \mathrm{~m}$ seaward in both spilling and plunging breakers. This is attributed to the fixed wind speed $(U / C=2)$ used here. It can be seen that the evolution of the wave shape has been slightly changed under the influence of wind. The wind also affects the shape of the overturning jet and the subsequent splash-up.
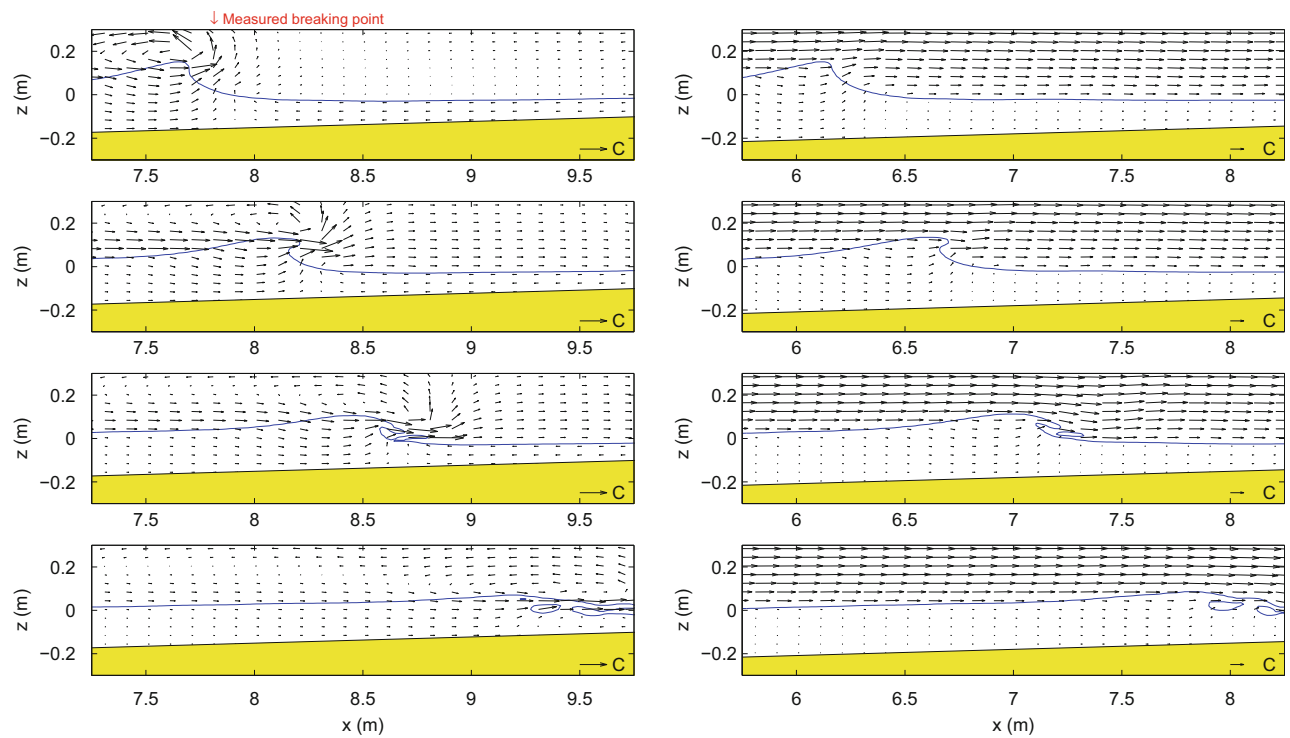

(a) $U / C=0$

(b) $U / C=2$

Fig. 7 Velocity fields during wave breaking for the plunging breaker case at $t / T=0.0,0.05,0.1,0.2$ when $\mathbf{a} U / C=0$ (from Xie 2013) and $\mathbf{b}$ $U / C=2$. Velocities are normalized by the wave phase speed $C$. For clarity, only velocity vectors in every five points are shown here 

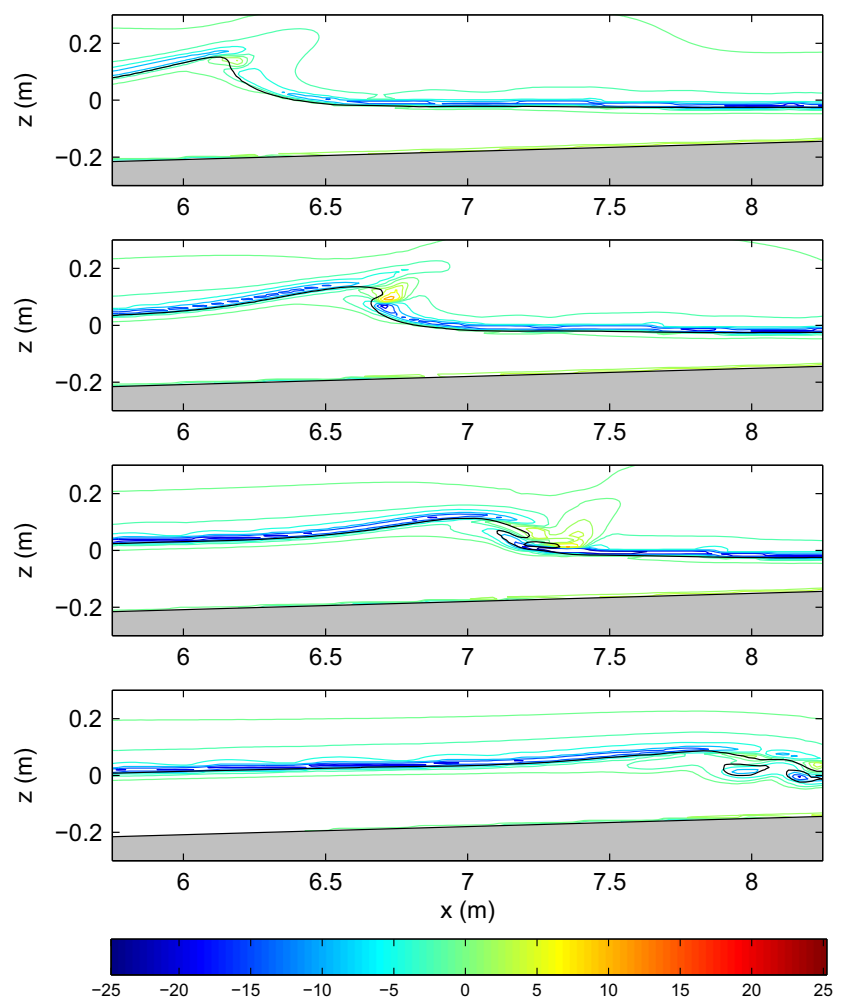

Fig. 8 Mean vorticity fields during wave breaking for the plunging breaker case when $U / C=2$ at $t / T=0.0,0.05,0.1,0.2$. The vorticity has been normalized by $\sqrt{g /\left(d_{0}+a\right)}$, where $a=\max [\zeta(x=$ $-4.3, t)]-d_{0}$ is the incident wave amplitude at the inlet and the contours are shown for $\pm[1,2.5,5,10,15,20,25]$

Figure 8 shows the mean vorticity fields for the plunging breaker when $U / C=2$ in one wave period at $t / T=$ $0.0,0.05,0.1,0.2$. In the air, similar to the spilling breaker, large negative vorticity is generated in the vicinity of the water surface due to the wind shear compared to the case for $U / C=0$ shown in Xie (2013). There is only negative vorticity in the air before wave breaking, whereas both the positive and negative vorticities coexist in front of the overturning jet after wave breaking. In the water, small negative vorticity is observed just underneath the water surface. However, the vorticity generation has been weakened in comparison with the case for $U / C=0$ as the region of negative vorticity becomes thinner and moves upward. This is because the wind affects the wave to break earlier in a deeper water, which leads to the change of the vortex motion after wave breaking. It is shown that vorticity generation is much stronger in the plunging breaker than that in the spilling breaker, especially during the jetsplash cycles. The negative vorticity is spread downward close to the bottom in the whole region underneath the wave front in plunging breakers (e.g., the vorticity contour of -2.5 goes down to $-0.04 \mathrm{~m}$ under the plunging breaker), whereas the vorticity is only confined to the region near the water surface in spilling breakers (e.g., the vorticity contour of -2.5 goes down to $-0.02 \mathrm{~m}$ under the spilling breaker).

Figure 9 shows the turbulent kinetic energy fields for the plunging breaker in one wave period at $t / T=0.0,0.05,0.1$, 0.2 with $(U / C=2)$ and without $(U / C=0)$ wind. Turbulence suppression by the wind can be observed here as the wave breaks in a deeper region, which is similar to spilling breakers. However, when compared to spilling breakers (Fig. 5), much higher production and spreading of turbulent kinetic energy occur in plunging breakers, especially in the jet-splash cycles.
Fig. 9 Turbulent kinetic energy fields during wave breaking for the plunging breaker case at $t / T=0.0,0.05,0.1,0.2$ when a $U / C=0$ and $\mathbf{b} U / C=2$. Turbulent kinetic energy are normalized by $C^{2}$
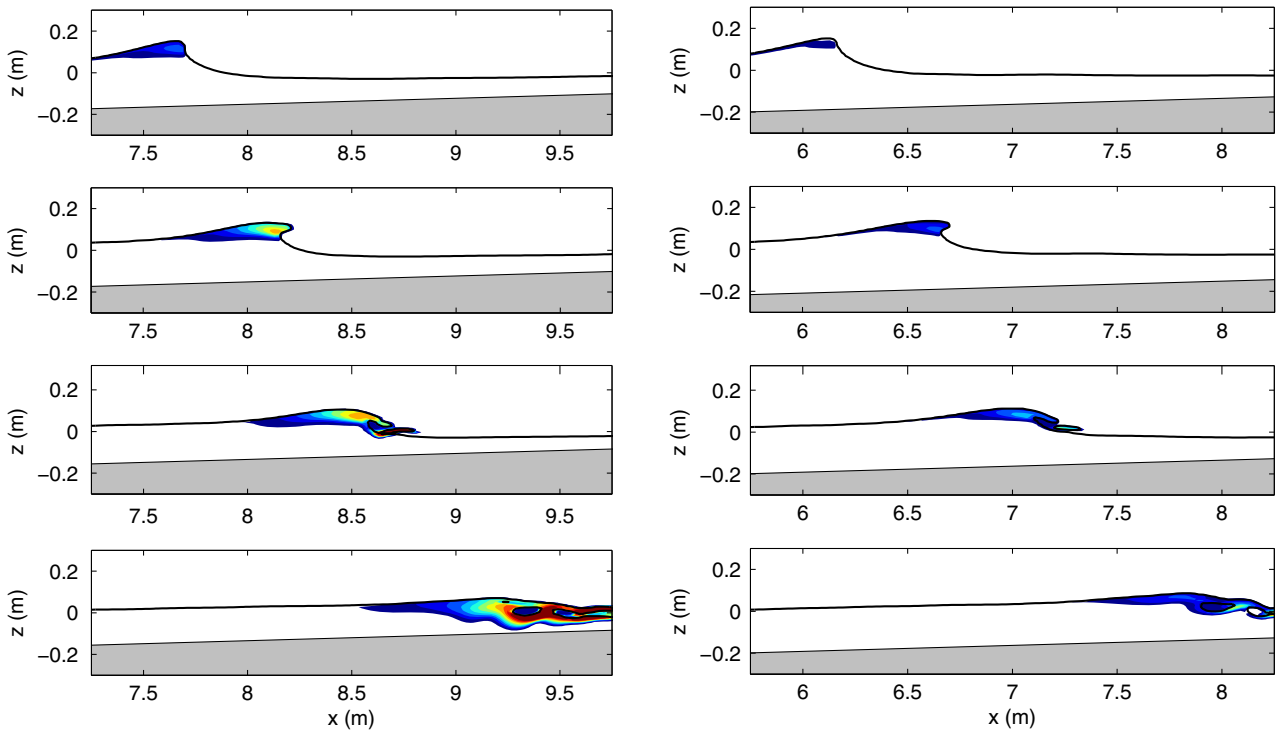

(a) $U / C=0$

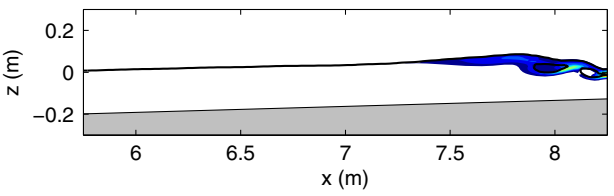

(b) $U / C=2$

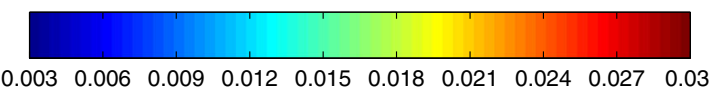




\section{Conclusions}

In this paper, we have investigated wind effects on periodic breaking waves in the surf zone, using a two-phase flow model. The model solves the RANS equations simultaneously for the flows both in the air and water, with the water surface calculated by the VOF method. The wave breaking, wave overturning and splash-up phenomena have been captured by the two-phase flow model, and the numerical results are in good agreement with previous experiments in the absence of wind (Xie 2013).

Detailed information of the effect of wind on both spilling and plunging breakers is given in this paper, focusing on the wave breaking process. Water surface profiles, velocity, vorticity and turbulence fields are shown and discussed. The wind effects on breaking waves in the surf zone obtained by the present numerical simulations are summarised as follows:

(i). Wind has a significant effect on the distribution of maximum wave elevation and mean water level in the surf zone. During wave shoaling, the maximum wave elevation is similar but occurs at different locations in the presence of wind.

(ii). In the presence of wind, the slope of the mean water level increases in comparison with the case in the absence of wind, which is attributed to wind forcing.

(iii). The breaker wave-height-to-water-depth ratio decreases in the presence of wind.

(iv). Wind affects the water surface profile during wave breaking and splash-up precesses. Onshore wind causes water waves to break earlier, in deeper water further from shore.

(v). The water particle velocity in breaking waves increases under the influence of onshore wind and thus affects the wave breaking process. There is a recirculation of air above the crest of the wave in the absence of wind while the air follows the wind flow in the presence of wind.

(vi). Wind increases the generation of vorticity near the air-water interface.

(vii). The turbulent kinetic energy is suppressed by the wind as the wave breaks in deeper water.

Acknowledgements ZX would like to acknowledge the financial support by the Marie Curie EST fellowship for his Ph.D. study at the University of Leeds and the Open Awards of the State Key Laboratory of Coastal and Offshore Engineering at Dalian University of Technology.

Open Access This article is distributed under the terms of the Creative Commons Attribution 4.0 International License (http:// creativecommons.org/licenses/by/4.0/), which permits unrestricted use, distribution, and reproduction in any medium, provided you give appropriate credit to the original author(s) and the source, provide a link to the Creative Commons license, and indicate if changes were made.

\section{References}

Bakhtyar R, Barry DA, Yeganeh-Bakhtiary A, Ghaheri A (2009) Numerical simulation of surf-swash zone motions and turbulent flow. Adv Water Resour 32(2):250-263

Banner ML (1990) The influence of wave breaking on the surface pressure distribution in wind wave interactions. J Fluid Mech 211:463-495

Banner ML, Peregrine DH (1993) Wave breaking in deep-water. Ann Rev Fluid Mech 25:373-397

Banner ML, Phillips OM (1974) Incipient breaking of small scale waves. J Fluid Mech 65:647-656

Battjes JA (1988) Surf-zone dynamics. Ann Rev Fluid Mech 20:257-293

Bonmarin P (1989) Geometric-properties of deep-water breaking waves. J Fluid Mech 209:405-433

Bradford SF (2000) Numerical simulation of surf zone dynamics. J Waterw Port Coast Ocean Eng-ASCE 126(1):1-13

Chen Q, Kaihatu JM, Hwang PA (2004) Incorporation of wind effects into Boussinesq wave models. JWaterw Port Coastal Ocean EngASCE 130(6):312-321

Christensen ED, Deigaard R (2001) Large eddy simulation of breaking waves. Coast Eng 42(1):53-86

Dias F, Kharif C (1999) Nonlinear gravity and capillary-gravity waves. Ann Rev Fluid Mech 31:301-346

Douglass SL (1990) Influence of wind on breaking waves. J Waterw Port Coastal Ocean Eng-ASCE 116(6):651-663

Duncan JH (1983) The breaking and non-breaking wave resistance of a two-dimensional hydrofoil. J Fluid Mech 126:507-520

Feddersen F, Veron F (2005) Wind effects on shoaling wave shape. J Phys Oceanogr 35(7):1223-1228

Hieu PD, Katsutohi T, Ca VT (2004) Numerical simulation of breaking waves using a two-phase flow model. Appl Math Modell 28(11):983-1005

Hirsch C (2007) Numerical computation of internal and external flows introduction to the fundamentals of CFD. new edn. ButterworthHeinemann, Oxford

Horikawa K (1988) Nearshore dynamics and coastal processes: Theory, measurement and predictive models. University of Tokyo Press, Tokyo

Issa RI (1986) Solution of the implicitly discretised fluid flow equations by operator-splitting. J Comput Phys 62(1):40-65

Jahne B, Haussecker H (1998) Air-water gas exchange. Ann Rev Fluid Mech 30:443-468

Jeffreys H (1925) On the formation of water waves by wind. Proc Royal Soc Lond Ser A-Contain Papers Math Phys Charact 107(742):189_ 206

Kharif C, Giovanangeli JP, Touboul J, Grare L, Pelinovsky E (2008) Influence of wind on extreme wave events: experimental and numerical approaches. J Fluid Mech 594:209-247

Kimmoun O, Branger H (2007) A particle image velocimetry investigation on laboratory surf-zone breaking waves over a sloping beach. J Fluid Mech 588:353-397

King DM, Baker CJ (1996) Changes to wave parameters in the surf zone due to wind effects. J Hydraul Res 34:55-76

Lakehal D, Liovic P (2011) Turbulence structure and interaction with steep breaking waves. J Fluid Mech 674:522-577

Launder BE, Spalding DB (1974) The numerical computation of turbulent flows. Comput Methods Appl Mech Eng 3(2):269-289

Lin JC, Rockwell D (1995) Evolution of a quasi-steady breaking wave. J Fluid Mech 302:29-44

Lin P (2008) Numerical Modeling of Water Waves: An introduction to engineers and scientists. Taylor \& Francis

Lin P, Liu PLF (1998a) A numerical study of breaking waves in the surf zone. J Fluid Mech 359:239-264 
Lin P, Liu PLF (1998b) Turbulence transport, vorticity dynamics, and solute mixing under plunging breaking waves in surf zone. $\mathrm{J}$ Geophys Res-Oceans 103(C8):15,677-15,694

Liu K, Chen Q, Kaihatu J (2016) Modeling wind effects on shallow water waves. Journal of Waterway, Port, Coastal, and Ocean Engineering-ASCE p 04015012

Longuet-Higgins MS, Stewart RW (1962) Radiation stress and mass transport in gravity waves, with application to surf beats. J Fluid Mech 13:481-504

Lubin P, Vincent S, Abadie S, Caltagirone JP (2006) Three-dimensional large eddy simulation of air entrainment under plunging breaking waves. Coast Eng 53(8):631-655

Lynett PJ (2006) Wave breaking velocity effects in depth-integrated models. Coast Eng 53(4):325-333

Madsen PA, Sorensen OR, Schaffer HA (1997) Surf zone dynamics simulated by a Boussinesq type model. Part I. Model description and cross-shore motion of regular waves. Coast Eng 32(4):255-287

Mayer S, Madsen PA (2000) Simulation of breaking waves in the surf zone using a Navier-Stokes solver. In: Proceedings of the 27th International Conference on Coastal Engineering, vol 1. ASCE, Sydney, Australia, pp 928-941

Mei CC, Liu PLF (1993) Surface-waves and coastal dynamics. Ann Rev Fluid Mech 25:215-240

Melville WK (1996) The role of surface-wave breaking in air-sea interaction. Ann Rev Fluid Mech 28:279-321

Melville WK, Veron F, White CJ (2002) The velocity field under breaking waves: coherent structures and turbulence. J Fluid Mech 454:203-233

Nadaoka K, Hino M, Koyano Y (1989) Structure of the turbulentflow field under breaking waves in the surf zone. J Fluid Mech 204:359-387

Peregrine DH (1983) Breaking waves on beaches. Ann Rev Fluid Mech 15:149-178

Perlin M, He JH, Bernal LP (1996) An experimental study of deep water plunging breakers. Phys Fluids 8(9):2365-2374

Rapp RJ, Melville WK (1990) Laboratory measurements of deepwater breaking waves. Philos Trans R Soc Lond Ser A-Math Phys Eng Sci 331(1622):735-800

Rodi W (1993) Turbulence models and their application in hydraulics : a state-of-the-art review, 3rd edn. Balkema, Rotterdam

Shao SD (2006) Simulation of breaking wave by SPH method coupled with k- $\epsilon$ model. J Hydraul Res 44(3):338-349

Shao SD, Ji CM (2006) SPH computation of plunging waves using a 2D sub-particle scale (SPS) turbulence model. Int J Numer Methods Fluids 51(8):913-936

Sobey RJ (1986) Wind-wave prediction. Ann Rev Fluid Mech 18:149_ 172
Sorensen RM (2006) Basic Coastal Engineering, 3rd edn. Springer, Berlin

Stansby PK, Feng T (2005) Kinematics and depth-integrated terms in surf zone waves from laboratory measurement. J Fluid Mech 529:279-310

Ting FCK, Kirby JT (1994) Observation of undertow and turbulence in a laboratory surf zone. Coast Eng 24(1-2):51-80

Torrey MD, Cloutman LD, Mjolsness RC, Hirt CW (1985) NASAVOF2D: a computer program for incompressible flows with free surfaces. Technical Report LA-10612-MS, Los Alamos Scientific Laboratory

Tulin MP, Waseda T (1999) Laboratory observations of wave group evolution, including breaking effects. J Fluid Mech 378:197232

Ubbink O (1997) Numerical prediction of two fluid systems with sharp interfaces. PhD thesis, Imperial College of Science Technology and Medicine, London, UK

Veeramony J, Svendsen IA (2000) The flow in surf-zone waves. Coast Eng 39(2-4):93-122

Versteeg HK, Malalasekera W (2007) An introduction to computational fluid dynamics : the finite volume method, 2nd edn. Pearson Prentice Hall, Harlow

Wang Z, Zou Q, Reeve DE (2009) Simulation of spilling breaking waves using a two phase flow CFD model. Comput Fluids 38(10):1995-2005

Ward DL, Wibner CG, Zhang J (1998) Runup on coastal revetments under the influence of onshore wind. J Coast Res 14(4):13251333

Watanabe Y, Saeki H, Hosking RJ (2005) Three-dimensional vortex structures under breaking waves. J Fluid Mech 545:291328

Xie Z (2010) Numerical modelling of breaking waves under the influence of wind. PhD thesis University of Leeds, Leeds, UK

Xie Z (2012) Numerical study of breaking waves by a two-phase flow model. Int J Numer Methods Fluids 70(2):246-268

Xie Z (2013) Two-phase flow modelling of spilling and plunging breaking waves. Appl Math Modell 37(6):3698-3713

Xie Z (2014) Numerical modelling of wind effects on breaking solitary waves. Eur J Mech / B Fluids 43:135-147

Xie Z (2015) A two-phase flow model for three-dimensional breaking waves over complex topography. Proc R Soc A: Math Phys Eng Sci 471:20150,101

Yan S, Ma QW (2010) Numerical simulation of interaction between wind and 2D freak waves. Eur J Mech - B/Fluids 29(1):18-31

Zhao Q, Armfield S, Tanimoto K (2004) Numerical simulation of breaking waves by a multi-scale turbulence model. Coastal Engineering 51(1):53-80 